\title{
XXIV.
}

Aus dem chirurgisch-poliklinischen Institute der Universität Leipzig (Director Prof. Friedrich).

\section{Zur Frage der Entstehung von Magengeschwüren und Leberinfarcten nach experimentellen Netzresectionen.}

\author{
Von \\ Dr. Eduard Sthamer, \\ Assistenzarzt.
}

Auf dem Chirurgencongress des Jahres 1899 hatte Freiherr v. Eiselsberg ${ }^{1}$ ) Mittheilung gemacht über einige Fälle von Magenund Duodenalblutungen nach Operationen am Netz, als deren Ursache sich in einigen Fällen Geschwürsbildungen nachweisen liessen. $\mathrm{Er}$ war auch dieser Frage experimentell näher getreten und hatte nach 4 resultatlosen Versuchen an Ratten bei einem Kaninchen nach $\mathrm{Ab}$ bindung und Torsion des Netzes Schleimhautblutungen im Magen nachweisen können. Auf dem nächstjährigen Congress wurde von Friedrich ${ }^{2}$ ) Bericht gegeben über Infaretbildungen in der Leber und Schleimhautnekrosen im Magen nach experimentellen Netzresectionen. Sein Schüler Hoffmann ${ }^{3}$ ), welcher die Friedrich'schen Versuche fortgesetzt hatte, referirte dann in seiner Dissertation des Genaueren über die bei den Versuchsreihen beobachteten Infarctbildungen in der Leber und Geschwürsbildungen im Magen.

Während v. E is els berg's Urtheil dahin ging, dass die Blutungen in die Magenschleimhaut als Folgen der Verschleppung von theils inficirten, theils wenig oder nicht inficirten Thromben aus der Operationsstelle aufzufassen seien, kamen Friedrich und $\mathrm{H}$ offmann auf Grund ihrer Versuche zu dem Ergebniss, dass die Infection der Thromben zur Nekrosenbildung in Leber und Magen nicht erforderlich sei, sondern dass die Nekrosen lediglich als Folgen der Circulationsstörung durch embolischen Gefässverschluss anzusehen seien.

1) v. Eiselsberg, Archiv f. klin. Chirurgie. Bd. LIX. S. 837.

2) Friedrich, Archiv f. klin. Chirurgie. Bd. LXI. Heft 4.

3) Hoffmann, Dissertation. Leipzig, Bruno Georgi. 1900. 
Engelhardt und Neck ${ }^{\mathrm{l}}$ ) haben danach in derselben Richtung Versuche angestellt und glaubten auf Grund derselben dieser Annahme Friedrich's von der aseptischen Entstehungsmöglichkeit der Nekrosen entgegentreten und die Infection als einen wesentlichen Factor bei der Entstehung der Magenulcerationen und Lebeninfarete ansehen zu mïssen.

Durch ihre histologischen Befunde haben die letztgenannten Autoren, namentlich durch den Nachweis von Embolien in Gefässen der Leber und des Magens den Beweis erbracht, dass nach Abbindung des Netzes thatsächlich in den genannten Organen Embolien zu Stande kommen und damit für die von v. Ei s el sberg für die Magenulcera, von Friedrich und $\mathrm{H}$ offmann auch für die Leberinfarete ausgesprochene Ansicht, dass diese Veränderungen auf embolische Verschliessungen von zur Ernährung wichtigen Gefässbahnen zurïckzuführen seien, eine weitere Stütze gebracht.

Auffallend ist es allerdings, dass Engelhardt und Neck in keinem ihrer 21 Versuche eine ausgesprochene Geschwürsbildung im Magen beobachten konnten, obgleich sie, wie gesagt, Thromben in den Gefässen nachzuweisen vermochten, während Friedrich und Hoffman n ganz ausgesprochene Geschwürsbildungen in einer ganzen Reihe von Fällen beobachten konnten.")

Es ist jedoch, wie von Friedrich und Hoffmann schon berichtet, bei deren Versuchsreihen mehrfach vorgekommen, dass bei gleichbleibender Technik in einer ganzen Anzahl von Fällen hintereinander die crwarteten Veränderungen ausblieben, wäbrend wieder in so und so viel Fällen hintereinander die Befunde positiv ausfielen. Eine befriedigende Erklärung, warum in der einen Reihe Infarcte und Ulcera zu Stande kamen, in der anderen nicht, konnte nicht gregeben werden.

Insbesondere muss der Versuch von Engelhardt und Neck, das Auftreten der Magenulcerationen von dem Vorhandensein von Bacterien in den Blutbahnen abhängig zu macherf, bei der hierseits eingehaltenen Versuchstechnik, wie sie seinerzeit von Friedrich und Hoffmann geübt und von mir auf meines Chefs Anregung erneut in einer grösseren Versuchsreihe, mit Rücksicht auf jene Engelhardt und Neck'sche Annahme hin, nachgeprüft worden ist, als ein durch S. 308 .

1) Engelhardt und Neck, Deutsche Zeitschrift f. Chirurgie. Bd. LVIII.

2) Vergl. Friedrich's Demonstration der Magenpräparate auf dem Chirurgencongress 1900 . 
ihre Experimente nicht ausreichend gestützter bezeichnet werden. Engelhardt und Neck schreiben wörtlich auf Seite 322:

„Für die Infection des Magens nach Netzabbindung lassen sich verschiedene Arten und Wege denken. Einmal kann es sich um eine rein bacteritische Ansiedelung in den Gefässen handeln, sei es auf dem Wege des venösen rückläufigen Transportes, sei es dass das Inficiens die Leber passirt, hier vielleicht, wie es in unserem Versuch (Nr. 20) geschah, bacteritische Nekrosen setzt, das Blut der Cava, des rechten Herzens, dann der Lungen und schliesslich das arterielle Blut und mit diesem den Magen inficirt."

Die Experimentatoren scheinen also als Ausgangspunkt der Infection die Operation, genauer gesagt, die ligirte Netzstelle anzusehen. Das wäre ja ganz gut denkbar und gegen eine Infection auf dem Wege des venösen rückläufigen Transportes wäre nichts einzuwenden, wenn man in jedem Falle von Magenulceration wenigstens Bacterien im Netzstumpf nachweisen könnte.

Den zweiten Erklärungsversuch halten wir aber für sehr unglücklich. Das „Inficiens", also Bacterien oder bacterienhaltiges Material, soll die Leber passiren, hier "bacteritische Nekrosen" setzen, durch Vena cava, Herz und Lungen gehen und, nachdem es dem arteriellen Kreislauf beigemischt worden ist, gerade in der Magenschleimhaut und nirgends anders und zwar in allen Versuchen wieder Nekrosen verursachen; das ist doch sehr unwahrscheinlich.

Die genannten Autoren fahren dann fort: „Ein Beispiel dieses Modus möchten wir in Versuch 20 erblicken."

Das Protokoll über diesen Versuch lautet:

„20. (Meerschweinchen.) Vollständige Abbindung des Netzes und Abtragung des unterbundenen Theiles. Der Tod war nach 2 Tagen eingetreten, nachdem sich das Thier nach der Operation wieder gut erholt hatte. Banchwunde ohne Reizung. Keine Zeichen von Peritonitis. Abimpfung aus der Bauchhölle ergiebt Colonien (kurze plumpe Stäbchen). Im Magen einzelne punktförmige Blutungen in der Schleimhaut. Leber: am rechten Leberlappenrand an einer Stelle ein grauweisser linsengrosser Herd. Netzstumpf verdickt.

Mikroskopisch: Bacteritische Lebernekrosen (kurze plumpe Stäbchen). Kleine Blutungen. In einzelnen abführenden Lebervenen ebenfalls kurze Stäbchen. Magen: Zahlreiche bacteritische Nekrosen (kurze plumpe Stäbchen). Die Nekrosen haben der Fläche nach gemessen einen Durchmesser bis zu $4 \mathrm{~mm}$, in den Venen des Netzstumpfes von Bacterien (Stäbchen) durchsetzte Thromben.

Dieser Versuch ist keineswegs einwandsfrei, und seine Ergebnisse sind daher nur mit grosser Vorsicht zu verwenden, denn das Thier ist, ohne dass die Section eine Todesursache ergeben hätte, zwei Tage 
nach der Operation eingegangen, ein Ereigniss, das uns nie vorgekommen ist, und das uns veranlasst, diesem Versuch skeptisch gegenüber zu stehen. Engelhardt und Neck schreiben zwar, die Obduction habe "kurz" nach dem Tode stattgefunden; nach dem ganzen Obductions- und bakteriologischen Befund kann man sich jedoch des Verdachts kaum erwehren, dass Leichenverïnderungen ${ }^{1}$ ) vorgelegen haben; denn darüber, dass Leicheninfectionen sehr schnell eintreten, kann nach neueren Untersuchungen kein Zweifel sein.

Aber selbst wenn die Obduction unmittelbar nach dem Tode stattgefunden hat, so ist schon die Thatsache, dass das Thier einer nicht genügend aufgeklärten Erkrankung erlegen ist, für uns ausreichend, den Versuch als nicht beweiskräftig anzusehen. Und dies ist der einzige Versuch, auf den Engelhardt und Neck ihre Annahme stiitzen.

Weshalb nehmen Engelhardt und Neck überhaupt an, dass es sich um eine von der Ligaturstelle ausgehende Infection handelt?

Schon Hoffmann's Versuch 16, bei dem ohne Operation lediglich durch Kneten des Leibes in tiefer bis zur $\Lambda$ sphyxie fortgesetzter Narkose Leberinfarcte auftraten, lätte Engelhardt und Neck überzeugen können, dass zum Zustandekommen eines Leberinfarctes eine embolische Infection nicht nöthig ist.

Wenn es num technisch gelingt, eine Infection von der Ligaturstelle aus absolut sicher auszuschalten, so bleibt theoretisch die Möglichkeit einer Infection von der Schleimhant des Magens aus bestehen. Diescr können wir aber nur eine secundäre Bedeutung beilegen, da die bakteritische Nekrose erst in Folge der durch die aseptische Embolie oder Thrombose hervorgerufenen Circulationsstörung. auftreten würde. Die Frage aber, wie weit die Nekrose der Magensehleimhant auf den peptischen Einfluss des Magensaftes und wie weit auf den Einfluss der in dem Magen enthaltenen Bakterien zuriickzufïhren ist, wollen wir hier unerörtert lassen, weil sie für unsere Untersuchungen zunächst belanglos ist.

Die Infectionsmöglichkeit an Orte der Entstehung fällt bei den Leberinfareten fort, da das normale Lebergewebe keine lebensfühigen Keime nachweisen lässt, wie wir uns auch noch in besonders hierauf gerichteten Versuchsreihen durch Verimpfung normalen Lebergewebes uiberzeugt haben. Bei den Infareten der Leber lässt sich also der

1) Ich will hier nur beiläufig bemerken, dass bei den von Friedrich, Ifof fm an n und mir angestellten Versuchen Leichenreränderungen ausgeschlossen sind, da immer dic Obduction sich unmittelbar an die Tödtung anschloss.

Dentsehe Zeitschrift f. Chirurgie. LXI. Bd. 
strikte Beweis führen, dass sie a septisch sind, sobald es gelingt, die Infarcte steril zu verimpfen.

Engelhardt und Neck haben selbst bei Infarcten, z. B. bei ihrem Versuch 6, in ihren Protokollen die Notiz gegeben: "Keine Bakterien". Wenn sie die Pruifung durch Verimpfung von Infarettheilen gemacht haben, so haben sie selbst schon den Beweis erbracht, dass Bakterien zur Entstehung der Infarcte nicht nöthig sind.

Diese Prüfung ist aber nothwendig, um mit Sicherheit die Betheiligung von Bakterien auszuschliessen, Schnittfärbungen auf Bakterien, die wir ebenfalls, stets mit negativem Resultat vorgenommen haben, und Abimpfungen mit der Platinöse durch einfachen Abstrich genügen hier nicht.

Die Angabe Engelhardt's und Neck's, dass sie bei Abimpfung aus der Peritonealhöhle Bakteriencolonieen erhielten, genügt jedenfalls nicht, um zu beweisen, dass diese auch vor der Eröffnung des Peritoneums bei der Section vorhanden gewesen sind, wenn nicht die Obductionstechnik angegeben wird, auf die hier ja ausserordentlich viel ankommt.

Wir haben bei sehr sorgfältig ausgearbeiteter Technik, die wir aus gedachten Gründen unten ausfülrlich erörtern werden, in keinem Falle Bakterien aus der freien Peritonealhöhle gewinnen können; wenn sie aufgetreten wären, würden wir keinen Anstand nehmen, sofort die Möglichkeit eines Versuchsfehlers oder einer Luftkeiminfection zuzugeben.

Alle diese Erwägungen haben uns veranlasst, nochmals der Frage der Bakterienbetheiligung bei der Entstehnng der Leberinfarcte und Magengeschwüre nachzugehen.

Die Fragestellung war eine einfache:

Sind Bakterien zur Entstehung der Infarete und Magenulcera nöthig?

Um diese Frage zu beantworten, versuchten wir unter sorgfältigster bakteriologischer Controlle die Ligatur und Resection des Netzes absolut steril auszuführen und bei der Obduction unter Vermeidung von Nebeninfectionen die Infarcte und Netzstümpfe steril anf flüssige und feste Nährböden zu verimpfen.

Bei der Operation wurde die bakteriologische Controlle lediglich durch Abimpfung aus der Peritonealhöhle gleich nach der Eröffnung. und kurz vor Schluss der Abdominalhöhle mit der Platinöse, sowie durch Einbringen von Theilen der zur Ligatur verwandten Seidenfäden in Bouillon ansgeführt; die Röhrchen wurden im Thermostaten bei $37^{\circ}$ gehalten. 
Bei der Obduction wurde nach aseptischer Eröffnung der Bauchhöhle sofort mittelst Platinöse aus der Peritonealhöhle abgeimpft, und dann der Netzstumpf sowie etwaige Leberinfarcte ebenfalls in Bouillonröhrchen gebracht.

So einfach der Operationsweg und die Controlle absolut sterilen Vorgehens zu sein scheinen, so verlangen sie doch eine grosse Sorgfalt und Aufmerksamkeit. Es ist mir selbst erst nach einer ganzen Reihe von Misserfolgen gelungen, die Technik in jeder nur wünschenswerthen Weise einwandsfrei exact zu gestalten ${ }^{1}$ ), sodass Nebeninfectionen, soweit wie möglich, ausgeschlossen waren. Am leichtesten passirt es bekanntlich bei der Herausnahme und dem Einbringen von Organtheilen, dass Nebeninfectionen eintreten.

Da es mit unseren bisherigen Sterilisationsverfahren nicht möglich ist, die menschliche Haut und ebensowenig die des Thieres absolut keimfrei zu machen, so haben wir zu vermeiden versucht, dass irgend ein Contact der Haut des Thieres oder der Hand des Operateurs mit der Operationswunde stattfinden konnte.

Ligatur- und Nahtseide wurde selbstverständlich nur frisch gekocht verwandt und die Sterilität der Seidenfäden immer gleichzeitig durch Verimpfung von Ligaturtheilen geprift.

Ieh will hier bemerken, dass an der Friedrich'schen Poliklinik zur Unterljindung und Nahi ausschliesslich gedrehte englische Seide benutzt wird, welche auf einen bügelartig umgebogenen Metallstab aufgewickelt durch 3 maliges 20 Minuten anhaltendes Kochen in Wasser sterilisirt wird.

Bei jeder Unterbindung wird - auch bei Operationen am Mensehen - sorefältig vermieden, den Theil des Fadens, der in der Wunde liegen bleiben soll, mit den Fingern zu berühren. Beim Schlingen und Anziehen wird der Faden möglichst an den Enden gefasst, sodass ein absolut keimfreies Versenken des Unterbindungsmaterials garantirt wird.

Die Operationstechnik gestaltete sich in den Versuchen folgendermaassen :

Dem auf ein Brett gefesselten Thier wird die Bauchhaut rasirt und mit Scife und heissem Wasser gereinigt; nach Abspulung der Seife wird nach der an der Chirurgischen Universitäts-Poliklinik üb-

1) Herrn Cand. med. Krïgel', der mich mit grosser Sorgfalt und steter Hülfsbereitschaft bei der Ausführung der Versuche unterstützte, möchte ich auch hier nochmals danken. 
lichen Technik das Operationsfeld noch mit Aether gründlich abgerieben.

Meist haben wir während der eigentlichen Operation mit Aether narkotisirt.

Operateur und Assistent sterilisiren sich sorgfältig. Die Umgebung des Operationsgebietes wird durch ein sterilisirtes Tuch, das den ganzen Tisch bedeckt und nur in der Mitte einen rechteckigen Ausschnitt von einer dem Operationsfeld entsprechenden Grösse hat, abgedeckt.

Jedes Instrument gilt nach einmaligem Gebrauch als inficirt und wird neu in Sodalösung gekocht. Neben der Linea alba wird die Bauchhaut auf jeder Seite durch eine Seidennaht angeschlungen, an der die Wundränder nach dem Hautschnitt durch den Assistenten emporgezogen werden. Da die Fäden zur Hälfte die Haut passirt haben, gelten sie als nicht mehr steril und werden vom Assistenten mittelst Gefässzangen gehalten.

Der Hautschnitt verläuft in der Mediane, dicht oberhalb des Processus ensiformis beginnend, $4 \mathrm{~cm}$ nach hinten. Ein Wundsperrhaken, ähnlich dem von Bo se angegebenen, hält die Hautränder, die ausserdem an den Fäden hochgezogen werden, auseinander. Nun folgt die Eröffnung der Bauchböble mit frischem Messer in der Linea alba für eine Ausdehnung von 1 bis $2 \mathrm{~cm}$. Es ist uns immer gelungen, durch diese kleine Oeffnung das Netz zu entwickeln und auf einen sterilen Tupfer bis zur Art. gastro-epiploica inf. auszubreiten, mit Gefässzangen zu fassen und unter Schonung der genannten Arterie abzubinden und zu reseciren. Das Pankreas ist immer geschont worden. Falls die Reposition des Netzstumpfes oder ausgepresster Darmtheile durch Unruhe des Thieres erschwert ist, werden beide Zeigefinger mit friseh gekochten Gummifingern bedeckt und mit diesen die Reposition vorgenommen.

Darauf wird Peritoneum und Musculatnr mit 2 bis 3 Knopfnähten geschlossen und die Haut in zweiter Schicht darüber durch fortlaufende Naht für sich vernäht.

Einen Verband haben wir zuletzt nicht mehr angelegt, da mehrfach unter Gazecollodiumverbïnden kleine Hautnekrosen eintraten, die übrigens auf den sonstigen Verlauf ohne Einfluss blieben. Die Heilung der Bauchwunde war in allen Fällen reactionslos.

Die Tödtung und die sofort angeschlossene Obduction wurde zwischen dem 4. und 9. Tag vorgenommen.

Das wieder auf demselben Brett gefesselte Thier wird durch Scheerenschlag (Durchschneidung des Halses) getödtet. Die vorher in weitester Ausdehnung auch seitlich geschorene Bauchhaut wird mit 
Zur Frage der Entstehung von Magengeschwüren und Leberinfareten u. s. w. 525

\section{Uebersichtstabelle der Versuchsergebnisse.}

$\bigcirc$ bedeutet keimfrei im Culturversuch. $\quad x$ bedeatet Bakterienwachsthum im Culturversuch.

I. Aseptisch ausgeführte Netzresectionen.

\begin{tabular}{|c|c|c|c|c|c|c|c|c|c|c|}
\hline \multirow{3}{*}{$\begin{array}{l}\text { Thier } \\
\text { Nr. }\end{array}$} & \multirow{3}{*}{ 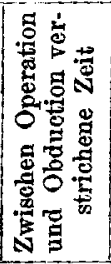 } & \multicolumn{7}{|c|}{ Abimpfungen bei } & \multirow[b]{3}{*}{ Anatomischer } & \multirow[b]{3}{*}{ Befund an } \\
\hline & & \multicolumn{3}{|c|}{ Operation } & \multicolumn{4}{|c|}{ Obduction } & & \\
\hline & & 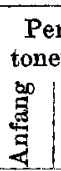 & 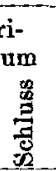 & 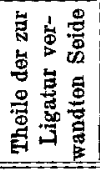 & 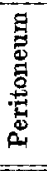 &  & 悹 & 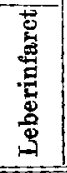 & & \\
\hline 1 & 7 Tage & 0 & 0 & 0 & & 0 & 0 & & - & 2 Ulcera. \\
\hline 2 & 7 Tage & 0 & 0 & 0 & & 0 & 0 & & - & - \\
\hline 3 & 7 Tage & 0 & 0 & 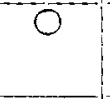 & 0 & 0 & 0 & 0 & $\begin{array}{c}\text { 1 zw eifelhafter, } \\
\text { hanfkorngrosser In- } \\
\text { farct. }\end{array}$ & $\cdots$ \\
\hline 5 & 5 Tage & & & 0 & 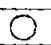 & $\overline{0}$ & & 0 & 1 Infaret. & - \\
\hline 6 & 5 Tage & 0 & 0 & 0 & 0 & 0 & & 0 & 4 Infarete. & - \\
\hline 7 & 5 Tage & 0 & & 0 & 0 & $x^{3}$ & & 0 & $\begin{array}{l}6 \text { Infarcte, } \mathrm{z} . \text { Th. } \\
\text { streifenförmig. }\end{array}$ & - \\
\hline 10 & 8 Tage & & 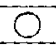 & 0 &  & 0 & & & - & - \\
\hline 14 & 5 Tage & 0 & 0 & $\left.x^{2}\right)$ & 0 & 0 & & & - & $=$ \\
\hline 15 & 5 Tage & 0 & 0 & 0 & 2 & 0 & & & - & $\begin{array}{l}3 \text { Ulcera, mehrfach } \\
\text { Hämorrhagien. }\end{array}$ \\
\hline 16 & 5 Tage & &  & 0 & $y$ & $x^{3}$ & & $\left.x^{3}\right)$ & 1 Infarct. & - \\
\hline 17 & 5 Tage & 0 & 0 & 0 & 0 & 0 & & 0 & 2 Infarcte. & - \\
\hline 18 & 5 Tage & 0 & 0 & 0 & 0 & 0 & & 0 & 2 Infarcte. & 1 Ulcus. \\
\hline 19 & 5 Tage & 0 & 0 & 0 & 0 & 0 & & & - & - \\
\hline
\end{tabular}

II. Nicht aseptisch ausgeführte Netzresectionen

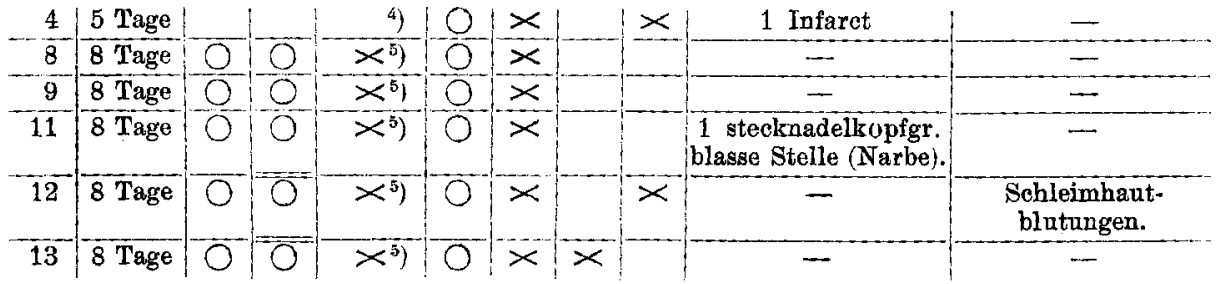

Bemerkungen:

1) Die Verimpfung des Netzstumpfes ergab Mikrokokken. Ein Versuchsfehler bei der Verimpfung ist verantwortlich zu machen.

2) Folge eines Versuchsfehlers.

3) Bei der Obduction wurde der Magen vorzeitig geöfnet, es trat Mageninhalt aus, und die Abimpiung gelang infolgedessen nicht aseptisch.

4) Die Operation wird ohne jede Asepsis ausgeführt, speciell die Seidenfäden mebrfach durch die nicht sterilisirten Finger gezogen.

5) In allen diesen Fällen wuchs Bacillus pyocyaneus sowohl in den bei der Operation als auch bei der Obduetion geimpften Röhrehen. Die Operation wurde aseptisch ausgeführt, nur ward nicht sterilisirte Seide zur Ligatur verwandt. 
dem Paquelinbrenner abgesengt und verschorft. Die Bauchhaut darauf mit sterilisirtem Messer hufeisenförmig, die Basis des Lappens nach dem Brustkorb zu, durchtrennt, und nun die Peritonealhöhle mit neuem Messer eröffnet und mit 2 Scheeren auf beiden Seiten entsprechend dem angelegten Hautschnitt durchtrennt. Es folgt die Abimpfung aus der Peritonealhöhle mittelst Platinöse.

Mit einer Muzeux'sehen Zange wird nun der Lappen etwas angehoben und unter demselben 1. die Ligaturstellen am Netz, 2. eventuelle Leberinfarcte mit frischer Scheere und Pincette abgetragen und in sterile Petrischälchen gebracht zur späteren Verarbeitung.

Jetzt ist der aseptische Theil der Obduction beendet.

Der Magen wird uneröffnet in Formalinlösung gebracht und die übrigen Organe ebenfalls secirt. Ich will gleich hier bemerken, dass wir nie Veränderungen an anderen Organen, speciell Niere, Milz und Lunge, nachweisen konnten.

Die Ligaturstellen des Netzes werden nun aus dem Petrischälchen in Bouillonröhrehen gebracht. Es versteht sich von selbst, dass das aseptische Arbeiten mit diesen kleinen, weichen, klebrigen Netztheilen Schwierigkeiten macht, sodass schon bei dem Einbringen in das Bouillonröhrchen die Möglichkeit einer Verunreinigung durch Luftkeime oft sehr wohl möglich erscheinen muss. In solchen Fällen wurden die Abimpfungen gleich als unsicher notirt.

Die Leberinfarcte wurden, wenn sie nicht zu klein waren oder bei der Herausnahme durch das Anfassen mit der Pincette zu sehr gelitten hatten, mit scharfem Messer getheilt und die eine Hälfte ebenfalls in Bouillon gebracht, während die andere zur mikroskopischen Untersuchung eingelegt wurde.

Das Resultat der Verimpfung wurde durch erneute Abimpfung nach 24-48 Stunden auf schräg erstarrten Agar und auf Bouillon controllirt. Oft, namentlich bei Leberinfareten, zeigte sich durch Sterilbleiben der zweiten Abimpfung, dass die im ersten Röhrchen entstandene Trübung nicht auf Bakterienentwicklung, sondern auf Auslaugung des Gewebsstückes zu beziehen war.

Aus unserer tabellarischen Zusammenstellung (S. 525) ergiebt sich nun, dass bei absolut aseptischem Verlauf in 3 Fällen (Fall 1, 15 und 18) Magengeschwüre auftraten. Leberinfarcte waren relativ häufiger. Mikroskopisch sicher gestellt und völlig aseptisch waren sie in 4 Fällen (5, 6, 7 und 18): Im Falle 18 traten also sowohl am Magen wie an der Leber Geschwüre resp. Infarcte auf.

Im Falle 7 war die Netzstumpfverimpfung nicht steril geblieben. Hier liegt wohl ein Versuchsfehler vor. Der Leberinfaret, der eben- 
falls verimpft wurde, blieb steril. Im Fall 16 wurde bei der Obduction der Magen versehentlich vorzeitig geöffnet, sodass gleich die Abimpfungen als zweifelhafte im Protokoll vermerkt wurden.

In den Fällen 8 bis 13 wurde nicht sterilisirte Seide zur Unterbindung verwandt. Es wuchs in 5 Fällen Bacillus pyocyaneus, sowohl bei Abimpfung der Fäden als auch bei Verimpfung der Netzstïmpfe und Leberstiicke. Gerade diese Versuchsreihe zeigt, dass trotz sicheren Vorhandenseins von Bakterien die Veränderungen an Magen und Leber nicht zahlreicher waren, als in den ganz aseptischen Fällen.

Die Diagnose der Infarete und Magenulcera wurde durch mikroskopische Untersuchung gesichert. Bei den Fällen, wo es in Folge der Kleinheit der Objecte nicht gelang, die Infarcte zur Verimpfung. und Untersuchung zu theilen, haben wir die Verimpfung vorgezogen und die nöthige Notiz im Protokoll eingetragen.

Die Nährböden wurden von uns selbst hergestellt und wiederholt auf ihre Eigenschaften, namentlich Alkalescenzgrad, controllirt.

Das Resultat unserer Untersuchungen ist, kurz zusammengefasst. das folgende:

Es treten beim Meerschweinchen nach völlig aseptisch ausgeführter Ligatur und Resection des Netzes Infarcte in der Leber und Geschw ürsbildung im Magen auf, ohne dass bei der Obduction Bakterienwachsthum aus der Ligaturstelle sowohl, wie aus den Infarctherden. nachgewiesen werden kann.

Wir ziehen daraus den Schluss, dass das Auftreten von Leberinfareten und Magenulcerationen nicht durch die Anwesenheit von Bakterien bedingt sein muss.

Der Einwand, dass es sich um Bakterien handeln könnte, die auf den gebräuchlichen Nährböden sich nicht züchten lassen (z. B. Anaëroben) wird insoweit hinfällig, als Engelhardt und Neck die Cultivirung der angeblich in Frage kommenden Bakterien ohne Weiteres gelungen ist. 